Pacific Journal of Mathematic 


\title{
ON GENERALIZED SUBHARMONIC FUNCTIONS
}

\author{
L. K. JACKSON
}

1. Introduction. In a previous paper [1], the notion of subharmonic functions was generalized in a manner corresponding to Beckenbach's [2] generalization of convex functions. This generalization was accomplished by replacing the dominating family of harmonic functions by a more general family of functions. In [1] the discussion was restricted to continuous subfunctions.

In the present paper we shall give some further properties of the dominating functions and extend the definition of subfunctions to permit upper semicontinuous subfunctions. We shall then show that results of J. W. Green [3] on approximately subharmonic functions extend to our subfunctions.

2. $\{F\}$ functions and $\operatorname{sub}-\{F\}$ functions. Let $D$ be a given plane domain and let $\{\gamma\}$ be a given family of contours bounding subdomains $\Gamma$ of $D$ such that $\bar{\Gamma}=\gamma+\Gamma \subset D$ where $\bar{\Gamma}$ indicates the closure of $\Gamma$. We assume that $\{\gamma\}$ contains all circles of radii less than a fixed number which lie, together with their interiors, in $D$. We shall use the Greek letter $\kappa$ to represent a circle of $\{\gamma\}$ and $K$ its interior. We shall use single small Roman letters to represent points in the plane. Let there be given a family of functions $\{F(x)\}$ which we shall call $\{F\}$-functions satisfying the following postulates.

Postulate 1. For any $\gamma \in\{\gamma\}$ and any continuous boundary value function $h(x)$ on $\gamma$, there is a unique $F(x ; h ; \gamma) \in\{F(x)\}$ such that

(a) $F(x ; h ; \gamma)=h(x)$ on $\gamma$,

(b) $\quad F(x ; h ; \gamma)$ is continuous in $\bar{\Gamma}$.

Postulate 2. If $h_{1}(x)$ and $h_{2}(x)$ are continuous on $\gamma$ and if $h_{1}(x)-$ $h_{2}(x) \leq M$ on $\gamma, M \geq 0$, then

$$
F\left(x ; h_{1} ; \gamma\right)-F\left(x ; h_{2} ; \gamma\right) \leq M
$$

Received August 1, 1953. This research was supported in part by a Summer Research Fellowship granted by the Research Council of the University of Nebraska.

Pacific J. Math. 5 (1955), 215 - 228 
in $\Gamma$; further, if the strict inequality holds at a point of $\gamma$, then the strict inequality holds throughout $\Gamma$.

Postulate 3. For any $\kappa \in\{y\}$ and for any collection $\left\{h_{\nu}(x)\right\}$ of functions $h_{\nu}(x)$ which are continuous and uniformly bounded on $\kappa$, the functions $F\left(x ; h_{\nu}\right.$; $\kappa)$ are equicontinuous in $K$.

Definition 1 . The function $s(x)$ is defined to be sub-\{F\} in $D$ provided

(a) $s(x)$ is bounded on every closed subset of $D$,

(b) $s(x)$ is upper semicontinuous in $D$,

(c) $s(x) \leq F(x)$ on $\gamma$ implies $s(x) \leq F(x)$ in $\Gamma$.

DEFINITION 2. The function $S(x)$ is defined to be super- $\{F\}$ in $D$ provided

(a) $S(x)$ is bounded on every closed subset of $D$,

(b) $S(x)$ is lower semicontinuous in $D$,

(c) $S(x) \geq F(x)$ on $y$ implies $S(x) \geq F(x)$ in $\Gamma$.

Let $\Omega$ be a bounded connected open set comprised together with its boundary $\omega$ in $D$ and let $g(x)$ be a bounded function defined on $\omega$.

DEFINITION 3. The function $\phi(x)$ is an under-function (relative to $g(x)$ ) if $\phi(x)$ is continuous in $\bar{\Omega}$, sub- $\{F\}$ in $\Omega$, and $\phi(x) \leq g(x)$ on $\omega$.

DEFINITION 4. The function $\psi(x)$ is an over-function (relative to $g(x)$ ) if $\psi(x)$ is continuous in $\bar{\Omega}$, super- $\{F\}$ in $\Omega$, and $\psi(x) \geq g(x)$ on $\omega$.

Postulate 4. If $\Omega$ is any bounded connected open set comprised together with its boundary $\omega$ in $D$ and $g(x)$ any bounded function defined on $\omega$, then the associated families of over-functions and under-functions are both non-null.

Postulate 5. For any circle $\kappa \in\{\gamma\}$ and any real number $M$, there exist continuous functions $h_{1}(x)$ and $h_{2}(x)$, defined on $\kappa$, such that

$$
F\left(x ; h_{1} ; \kappa\right) \geq U, \quad F\left(x ; h_{2} ; \kappa\right) \leq M \quad \text { in } \bar{K} .
$$

Postulate 6. For any circle $\kappa \in\{\gamma\}$, if the functions $h_{n}(x)(n=0,1,2, \ldots)$, defined on $\kappa$, are continuous and uniformly bounded on $\kappa$, and

$$
\lim _{n \rightarrow \infty} h_{n}(x)=h_{0}(x)
$$


for all but at most a finite number of points of $\kappa$, then

$$
\lim _{n \rightarrow \infty} F\left(x ; h_{n} ; \kappa\right)=F\left(x ; h_{0} ; \kappa\right)
$$

at all points of $K$.

Postulate 7. For any circle $\kappa \in\{\gamma\}$, if the functions $h_{n}(x)(n=1,2, \cdots)$, defined on $\kappa$, are continuous and uniformly bounded on $\kappa$ and equicontinuous at a point $x_{0} \in \kappa$, then the functions $F\left(x ; h_{n} ; \kappa\right)(n=1,2, \cdots)$, defined in $\bar{K}$, are equicontinuous at $x_{0}$.

Our definition of sub- $\{F\}$ functions differs from the definition of subharmonic functions in that we have restricted our subfunctions to be bounded on closed subsets of $D$. This seems to be necessary since we do not have a Harnack theorem of the type that is available in the theory of harmonic functions.

\section{Some theorems concerning the $\{F\}$-functions.}

THE OREM 1. If $\kappa \in\{\gamma\}, N$ is any real number, and $x_{0} \in K$, then there exists a continuous function $h(x)$ defined on $\kappa$ such that $F\left(x_{0} ; h ; \kappa\right)=N$.

Proof. By Postulate 5 there exist continuous functions $h_{1}(x)$ and $h_{2}(x)$ defined on $\kappa$ such that

$$
F\left(x ; h_{1} ; \kappa\right)>N, F\left(x ; h_{2} ; \kappa\right)<N \text { on } \bar{K}
$$

We define on $\kappa$

$$
h_{\lambda}(x)=\lambda h_{1}(x)+(1-\lambda) h_{2}(x), \quad 0<\lambda<1 .
$$

Then for $0<\lambda<1$ we have

$$
F\left(x_{0} ; h_{2} ; \kappa\right)<F\left(x_{0} ; h_{\lambda} ; \kappa\right)<F\left(x_{0} ; h_{1} ; \kappa\right) .
$$

Now set

$$
\lambda_{1}=\text { g.l.b. }\left[\lambda \mid F\left(x_{0} ; h_{\lambda} ; \kappa\right) \geq N\right]
$$

and

$$
\lambda_{2}=\text { l.u.b. }\left[\lambda \mid F\left(x_{0} ; h_{\lambda} ; \kappa\right) \leq N\right] \text {. }
$$


Using Postulate 2 we see that $\lambda_{1}=\lambda_{2}$ and

$$
F\left(x_{0} ; h_{\lambda_{1}} ; \kappa\right)=N \text {. }
$$

This result shows that Postulate 6 of [1] is actually a consequence of Postulates 1, 2, and 5 and may be omitted.

THEOREM 2. If $f(x)$ is continuous in $D$ and $x_{0} \in D$, then given any $\epsilon>0$, there exists $\kappa \in\{\gamma\}$ with center at $x_{0}$ and radius arbitrarily small such that

$$
|F(x ; f ; \kappa)-f(x)|<\epsilon \text { in } \bar{K} .
$$

Proof. If $\kappa_{1}$ is any circle of $\{\gamma\}$ with center at $x_{0}$, then by Theorem 1 there exists a continuous function $h(x)$ defined on $\kappa_{1}$ such that

$$
F\left(x_{0} ; h ; \kappa_{1}\right)=f\left(x_{0}\right) \text {. }
$$

By continuity there exists a smaller concentric circle $\kappa$ such that

$$
\left|F\left(x ; h ; \kappa_{1}\right)-f(x)\right|<\epsilon / 2 \text { in } \bar{K} .
$$

Let

$$
\begin{aligned}
& h_{1}(x)=\max \left[F\left(x ; h ; \kappa_{1}\right), f(x)\right] \text { on } \kappa, \\
& h_{2}(x)=\min \left[F\left(x ; h ; \kappa_{1}\right), f(x)\right] \text { on } \kappa .
\end{aligned}
$$

Then in $\bar{K}$

$$
\begin{aligned}
& F\left(x ; h_{2} ; \kappa\right) \leq F\left(x ; h ; \kappa_{1}\right) \leq F\left(x ; h_{1} ; \kappa\right), \\
& F\left(x ; h_{2} ; \kappa\right) \leq F(x ; f ; \kappa) \leq F\left(x ; h_{1} ; \kappa\right), \\
& F\left(x ; h_{1} ; \kappa\right)-F\left(x ; h_{2} ; \kappa\right)<\epsilon / 2 .
\end{aligned}
$$

Therefore in $\bar{K}$ we have

$$
|F(x ; f ; \kappa)-f(x)| \leq\left|F(x ; f ; \kappa)-F\left(x ; h ; \kappa_{1}\right)\right|+\left|F\left(x ; h ; \kappa_{1}\right)-f(x)\right|<\epsilon .
$$

THE OREM 3. If $f(x)$ is bounded and upper semicontinuous on $\gamma$, then there exists a function $F(x ; f ; y)$ such that

(1) $F(x ; f ; \gamma)$ is upper semicontinuous in $\bar{\Gamma}$ and continuous in $\Gamma$,

(2) $F(x ; f ; \gamma)$ is an $\{F\}$-function in $\Gamma$, 
(3)

$F(x ; f ; \gamma)=f(x)$ on $\gamma$.

Proof. Let $F(x ; f ; \gamma)$ be the infimum in $\bar{\Gamma}$ of all over-functions in $\bar{\Gamma}$ with respect to the boundary function $f(x)$ on $\gamma$. Clearly $F(x ; f ; \gamma)$ is upper semicontinuous in $\bar{\Gamma}$. Theorems 11 and 12 of $[1]$ show that $F(x ; f ; y)$ is continuous and an $\{F\}$-function in $\Gamma$. Let $f_{n}(x)(n=1,2, \ldots)$ be a monotone decreasing sequence of continuous functions converging to $f(x)$ on $\gamma$. Then $F\left(x ; f_{n} ; \gamma\right)$ is an over-function for each $n$ and therefore $F(x ; f ; \gamma)=f(x)$ on $\gamma$.

Heretofore we have used the notation $F(x ; h ; \gamma)$ only for functions continuous in $\bar{\Gamma}$ but henceforth we shall use the same notation when $h(x)$ is bounded and upper semicontinuous on $\gamma$ and $F(x ; h ; \gamma)$ is defined as in Theorem 3. No confusion should arise since, for $h(x)$ continuous on $\gamma$, the $F(x ; h ; \gamma)$ as defined by Theorem 3 is the unique $F(x ; h ; \gamma)$ of Postulate 1 . Hence, if $s(x)$ is sub- $\{F\}$ in $D$ and $\gamma \in\{\gamma\}$, there exists an $\{F\}$-function $F(x ; s ; \gamma)$ such that

$$
s(x)=F(x ; s ; \gamma) \text { on } \gamma
$$

and

$$
s(x) \leq F(x ; s ; \gamma) \text { in } \Gamma \text {. }
$$

THEOREM 4. If $h_{1}(x)$ and $h_{2}(x)$ are bounded and upper semicontinuous on $\gamma$ and $h_{1}(x)-h_{2}(x) \leq M$ on $\gamma, M \geq 0$, then

$$
F\left(x ; h_{1} ; y\right)-F\left(x ; h_{2} ; \gamma\right) \leq M \text { on } \Gamma .
$$

Proof. Let $x_{0} \in \Gamma$ and suppose that

$$
F\left(x_{0} ; h_{1} ; \gamma\right)-F\left(x_{0} ; h_{2} ; \gamma\right)=M+\delta,
$$

By Postulate 4 and Theorem 3 there exists an over-function $\psi(x)$ with respect to $h_{2}(x)$ such that

$$
0 \leq \psi\left(x_{0}\right)-F\left(x_{0} ; h_{2} ; \gamma\right)<\delta
$$

Then $F(x ; \psi ; \gamma)$ is also an over-function with respect to $h_{2}(x)$ and

$$
0 \leq F\left(x_{0} ; \psi ; \gamma\right)-F\left(x_{0} ; h_{2} ; \gamma\right)<\delta \text {. }
$$

Furthermore $F(x ; \psi+M, \gamma)$ is an over-function with respect to $h_{1}(x)$; hence, by the preceding inequality and Postulate 2 we have 


$$
\begin{aligned}
& F\left(x_{0} ; h_{1} ; \gamma\right)-F\left(x_{0} ; h_{2} ; \gamma\right) \leq F\left(x_{0} ; \psi+M ; \gamma\right)-F\left(x_{0} ; h_{2} ; \gamma\right) \\
& \quad \leq F\left(x_{0} ; \psi+M ; \gamma\right)-F\left(x_{0} ; \psi ; \gamma\right)+F\left(x_{0} ; \psi ; \gamma\right)-F\left(x_{0} ; h_{2} ; \gamma\right)<M+\delta .
\end{aligned}
$$

This is a contradiction and the theorem is proved.

THEOREM 5. If the functions $\left\{h_{\nu}(x)\right\}$ are upper semicontinuous and uniformly bounded on $\kappa$, then the functions $F\left(x ; h_{\nu} ; \kappa\right)$ are equicontinuous in $K$; further the function

$$
u(x) \equiv \sup _{\nu} F\left(x ; h_{\nu} ; \kappa\right)
$$

is continuous and $s u b-\{F\}$ in $K$, and

$$
v(x) \equiv \inf _{\nu} F\left(x ; h_{\nu} ; \kappa\right)
$$

is continuous and super-\{F\} in $K$.

The proof follows immediately from Postulate 3 and Theorem 4, and Lemma 1 and Theorem 11 of $[1]$.

\section{Some properties of $\operatorname{sub}-\{F\}$ functions.}

THE OREM 6. A necessary and sufficient condition for the function $s(x)$, which is upper semicontinuous in $D$ and bounded on every closed subset of $D$, to be sub-\{F\} in $D$ is that corresponding to each $x_{0} \in D$ there exists a sequence of circles $\kappa_{n}$ with centers at $x_{0}$ and radii $r_{n}\left(x_{0}\right) \rightarrow 0$ such that

$$
s\left(x_{0}\right) \leq F\left(x_{0} ; s ; \kappa_{n}\right)
$$

for each $n$.

THE OREM, 7. If $s_{1}(x), \cdots, s_{n}(x)$ are $s u b-\{F\}$ in $D$, then

$$
s(x)=\max \left[s_{1}(x), \cdots, s_{n}(x)\right]
$$

is $s u b-\{F\}$ in $D$.

THEOREM 8. If $s(x)$ is $s u b-\{F\}$ in $D$ and $\gamma \in\{\gamma\}$, then

$$
s(x ; y) \equiv \begin{cases}s(x) & \text { for } x \in D-\bar{\Gamma} \\ F(x ; s ; y) & \text { for } x \in \bar{\Gamma}\end{cases}
$$


is $s u b-\{F\}$ in $D$.

The proofs of these theorems parallel those given for continuous sub- $\{F\}$ functions in [1] and will be omitted.

THE OREM 9. If $s(x)$ is $s u b-\{F\}$ in $D$, then $s(x)-M, M \geq 0$, is $\operatorname{sub}-\{F\}$ in $D$.

Proof. Since $s(x)$ is upper semicontinuous in $D$ and bounded on every closed subset of $D, s(x)-M$ has the same properties. Now let $x_{0} \in D$ and $\kappa \in\{\gamma\}$ have its center at $x_{0}$. Then by Theorem 4

$$
s\left(x_{0}\right) \leq F\left(x_{0} ; s ; \kappa\right) \leq M+F\left(x_{0} ; s-M ; \kappa\right),
$$

hence,

$$
s\left(x_{0}\right)-M \leq F\left(x_{0} ; s-M ; \kappa\right)
$$

and by Theorem $6 s(x)-M$ is sub- $\{F\}$ in $D$.

\section{A Harnack theorem for the $\{F\}$-functions.}

THEOREM 10. If the decreasing sequence of $s u b-\{F\}$ functions $\left\{s_{n}(x)\right\}$ is uniformly bounded on each closed subset of $D$, then

$$
\lim _{n \rightarrow \infty} s_{n}(x)=s(x)
$$

is $\operatorname{sub}-\{F\}$ in $D$.

Proof. Clearly $s(x)$ is upper semicontinuous and bounded on every closed subset of $D$; hence, to show that $s(x)$ is $\operatorname{sub}-\{F\}$ in $D$ it will be sufficient to show that it satisfies the Littlewood criterion of Theorem 6 .

Let $x_{0} \in D$ and let $\kappa \in\{\gamma\}$ have its center at $x_{0}$. By Theorem 4 we have

$$
F\left(x ; s_{n+1} ; \kappa\right) \leq F\left(x ; s_{n} ; \kappa\right)
$$

and

$$
F(x ; s ; \kappa) \leq F\left(x ; s_{n} ; \kappa\right) \text {, in } \bar{K}
$$

for each $n$. Since $s_{n}(x)$ is $\operatorname{sub}-\{F\}$ in $D$ for each $n$ and the sequence $\left\{s_{n}(x)\right\}$ is decreasing, it follows that 


$$
s\left(x_{0}\right) \leq s_{n}\left(x_{0}\right) \leq F\left(x_{0} ; s_{n} ; \kappa\right) .
$$

Therefore,

$$
s\left(x_{0}\right) \leq \lim _{n \rightarrow \infty} F\left(x_{0} ; s_{n} ; \kappa\right),
$$

and we conclude the proof by showing that

$$
\lim _{n \rightarrow \infty} F\left(x_{0} ; s_{n} ; \kappa\right)=F\left(x_{0} ; s ; \kappa\right) .
$$

Since

$$
\lim _{n \rightarrow \infty} F\left(x_{0} ; s_{n} ; \kappa\right) \geq F\left(x_{0} ; s ; \kappa\right)
$$

assume

$$
\lim _{n \rightarrow \infty} F\left(x_{0} ; s_{n} ; \kappa\right)=F\left(x_{0} ; s ; \kappa\right)+\delta, \quad \delta>0
$$

There exists an over-function $\psi(x)$ with respect to the boundary function $s(x)$ on $\kappa$ such that

$$
F\left(x_{0} ; s ; \kappa\right) \leq \psi\left(x_{0}\right)<F\left(x_{0} ; s ; \kappa\right)+\delta / 2 \text {. }
$$

Since $\psi(x)$ is super- $\{F\}$ in $K$ we have

$$
F\left(x_{0} ; s ; \kappa\right) \leq F\left(x_{0} ; \psi ; \kappa\right)<F\left(x_{0} ; s ; \kappa\right)+\delta / 2 \text {. }
$$

An application of Postulate 2 then gives

$$
F\left(x_{0} ; s ; \kappa\right)<F\left(x_{0} ; \psi+\delta / 4 ; \kappa\right)<F\left(x_{0} ; s ; \kappa\right)+3 \delta / 4 .
$$

Since $\psi(x)+\delta / 4$ is continuous on $\kappa$ and

$$
s(x)<\psi(x)+\delta / 4
$$

on $\kappa$, it follows that for $N$ sufficiently large we have

$$
s_{n}(x)<\psi(x)+\delta / 4
$$

on $\kappa$ for $n \geq N$. Then for $n \geq N$

$$
F\left(x_{0} ; s_{n} ; \kappa\right) \leq F\left(x_{0} ; \psi+\delta / 4 ; \kappa\right)<F\left(x_{0} ; s ; \kappa\right)+3 \delta / 4 .
$$


This is a contradiction, hence

$$
\lim _{n \rightarrow \infty} F\left(x_{0} ; s_{n} ; \kappa\right)=F\left(x_{0} ; s ; \kappa\right)
$$

and

$$
s\left(x_{0}\right) \leq F\left(x_{0} ; s ; \kappa\right)
$$

Consequently by Theorem $6 s(x)$ is $\operatorname{sub}-\{F\}$ in $D$.

As an immediate consequence of Theorem 5 and Theorem 10 we have the following Harnack type theorem for the $\{F\}$-functions:

THEOREM 11. The limit of a uniformly bounded monotone decreasing sequence of $\{F\}$-functions is an $\{F\}$-function.

Furthermore it is clear that if $f(x)$ is bounded and upper semicontinuous on $\kappa$, if $F(x ; f ; \kappa)$ is the $\{F\}$-function defined in Theorem 3 , and if $\left\{f_{n}(x)\right\}$ $(n=1,2, \ldots)$ is any monotone decreasing sequence of continuous functions converging to $f(x)$ on $\kappa$, then

$$
\lim _{n \rightarrow \infty} F\left(x ; f_{n} ; \kappa\right)=F(x ; f ; \kappa) \text { in } \bar{K}
$$

6. Approximately sub $\{F\}$ functions. D. II. Hyers and S. M. Ulam [4] have introduced the notion of approximately convex functions. A function $f(x)$ is said to be approximately convex provided

$$
f(\lambda x+(1-\lambda) y) \leq \epsilon+\lambda f(x)+(1-\lambda) f(y)
$$

for $0 \leq \lambda \leq 1$ and for a fixed $\epsilon>0$. For $\epsilon=0$ the definition is that of a convex function.

The notion of a subharmonic function may be thought of as an extension to two dimensions of the notion of a convex function in one dimension. Using this idea, Green [3] has defined an approximately subharmonic function as follows: a function $f(x)$ defined in a domain $D$ is $\epsilon$-subharmonic provided (a) it is upper semicontinuous, and (b) if $h(x)$ is a harmonic function in a domain $D^{\prime}$ interior to $L$, which is continuous on the boundary of $D^{\prime}$ and dominates $f(x)$ there on, then in $D^{\prime}$

$$
f(x) \leq \epsilon+h(x)
$$

In an analogous way we define an approximately sub- $\{F\}$ function as follows: 
Definition 5. A function $g(x)$ is said to be $\epsilon$-sub- $\{F\}$ in $D$ provided

(a) $g(x)$ is bounded on every closed subset of $D$,

(b) $g(x)$ is upper semicontinuous in $D$,

(c) $g(x) \leq F(x)$ on the boundary of a subdomain $D^{\prime}$ of $D$ implies $g(x) \leq \epsilon+F(x)$ in $D^{\prime}$.

With this definition the theorem of Green for approximately subharmonic functions extends to approximately sub- $\{F\}$ functions.

THE ORE M 12. If $g(x)$ is $\epsilon$-sub- $\{F\}$ in $D$, there exists a function $u(x)$, sub- $\{F\}$ in $D$, such that $u(x) \leq g(x) \leq \epsilon+u(x)$ in $D$.

The proof of the theorem depends on the existence of a maximal sub- $\{F\}$ minorant for a continuous function. We shall give the proof of Theorem 12 after we have considered this question.

7. Maximal sub- $\{F\}$ minorants. The theorem given in this section has the same statement as the corresponding theorem for subharmonic functions and the proof is similar to the one given in [3].

THEOREM 13. If $f(x)$ is continuous in a domain $R \subset D$ and has a sub-\{F\} minorant in $R$, then it has a maximal sub-\{F\} minorant $u(x)$. The function $u(x)$ is continuous in $R$ and is an $\{F\}$-function where it is less than $f(x)$.

Proof. Let $S$ be the family of all functions sub- $\{F\}$ in $R$ and dominated by $f(x)$. By hypothesis $S$ is non-null. For $x \in R$ we define

$$
u(x)=\sup _{s \in S} s(x) .
$$

We wish to show first that $u(x)$ is lower semicontinuous in $R$. Let $x_{0} \in R$ and $\eta>0$, then there exists $s(x) \in S$ such that

$$
u\left(x_{0}\right)-\eta \leq s\left(x_{0}\right) \leq u\left(x_{0}\right) \leq f\left(x_{0}\right) .
$$

Then $s\left(x_{0}\right)-\eta<f\left(x_{0}\right)-\eta / 2$ and, by the continuity of $f(x)$ and the upper semicontinuity of $s(x)$, there exists a circle $\kappa_{1}$ with center at $x_{0}$ such that

$$
s(x)-\eta<f\left(x_{0}\right)-\eta / 2<f(x) \text { in } \bar{K}_{1} .
$$

By Theorem 2 we may choose a circle $\kappa$ with center at $x_{0}$, with radius less than that of $\kappa_{1}$, and such that 


$$
F\left(x ; f\left(x_{0}\right)-\eta / 2 ; \kappa\right)<f(x) \text { in } \bar{K}
$$

Then by Theorem 4 we have also

$$
F(x ; s-\eta ; \kappa)<f(x) \text { in } \bar{K} .
$$

Now define

$$
s^{*}(x ; \kappa)= \begin{cases}s(x)-\eta & \text { for } x \text { in } R-\bar{K} \\ F(x ; s-\eta ; \kappa) & \text { in } \bar{K} .\end{cases}
$$

It follows from Theorem 8 and 9 that $s^{*}(x ; \kappa) \in S$. Therefore,

$$
\liminf _{x \rightarrow x_{0}} u(x) \geq \liminf _{x \rightarrow x_{0}} s^{*}(x ; \kappa)=F\left(x_{0} ; s-\eta ; \kappa\right) \geq s\left(x_{0}\right)-\eta
$$

hence,

$$
\lim _{x \rightarrow x_{0}} u(x) \geq u\left(x_{0}\right)-2 \eta \text {. }
$$

Since $\eta$ is arbitrary this implies that

$$
\lim _{x \rightarrow x_{0}} u(x) \geq u\left(x_{0}\right)
$$

and $u(x)$ is lower semicontinuous in $R$.

Now we designate by $A$ the set of all $x \in R$ such that $u(x)=f(x)$ and let $B=R n$ comp $\bar{A} . B$ is an open set and for the moment we assume that it is not void. Let $x \in B$, then since $B$ is open, there exists a circle $\kappa$ with center at $x$ such that $\bar{K} \subset B$. Suppose that there exists an $s(x) \in S$ such that

$$
F(x ; s ; \kappa) \geq f(x)
$$

at some points of $K$. Then by Postulate 5 and Theorem 4 there would exist an $\eta \geq 0$ such that

$$
F(x ; s-\eta ; \kappa) \leq f(x)
$$

in $\bar{K}$ with the equality holding at some points of $K$. If for this $s(x), \kappa$, and $\eta$ we again define

$$
s^{*}(x ; \kappa)= \begin{cases}s(x)-\eta & \text { for } x \text { in } R-\bar{K} \\ F(x ; s-\eta ; \kappa) & \text { in } \bar{K} .\end{cases}
$$


then $s^{*}(x ; \kappa) \in S$ and $s^{*}(s ; \kappa)=f(x)$ at some points of $K$. Thus we would have $u(x)=f(x)$ at some points of $K$ and this would contradict $\bar{K} \subset B$. Hence

$$
F(x ; s ; \kappa)<f(x) \text { in } \bar{K}
$$

for every $s(x) \in S$. Hence for every $s(x) \in S$ let

$$
s(x ; \kappa)= \begin{cases}s(x) & \text { in } R-\bar{K} \\ F(x ; s ; \kappa) & \text { in } \bar{K} .\end{cases}
$$

Then $s(x ; \kappa) \in S$ and $s(x) \leq s(x ; \kappa)$ in $R$, therefore,

$$
u(x)=\sup _{s \in S} s(x)=\sup _{s \in S} s(x ; \kappa) .
$$

We conclude by Theorem 5 that $u(x)$ is continuous and sub-\{F\} in $K$ and hence in $B$.

Now we define

$$
u^{*}(x)= \begin{cases}f(x) & \text { for } x \in \bar{A} \\ u(x) & \text { for } x \in B\end{cases}
$$

Then clearly $u^{*}(x)$ is upper semicontinuous in $R$ and $u(x) \leq u^{*}(x) \leq f(x)$ in $R$.

Next we show that $u^{*}(x)$ is sub- $\{F\}$ in $R$. We have already observed that $u(x)$ is $\operatorname{sub}-\{F\}$ in $B$, hence $u^{*}(x)$ is $\operatorname{sub}\{F\}$ in $B$. Let $x_{0} \in \bar{A}$ and let $\kappa \in\{\gamma\}$ have its center at $x_{0}$ and $\bar{K} \subset R$, then

$$
s(x) \leq F(x ; s ; \kappa) \leq F\left(x ; u^{*} ; \kappa\right) \text { in } \bar{K}
$$

for every $s(x) \in S$. For $x \in A \cap K$

$$
\sup _{s \in S} s(x)=f(x) \leq F\left(x ; u^{*} ; \kappa\right) ;
$$

it follows by the continuity of $f(x)$ and $F\left(x ; u^{*} ; \kappa\right)$ in $K$ that

$$
u^{*}\left(x_{0}\right)=f\left(x_{0}\right) \leq F\left(x_{0} ; u^{*} ; \kappa\right) .
$$

By Theorem 6, $u^{*}(x)$ is sub- $\{F\}$ in $R$, therefore $u^{*}(x) \in S$ and $u^{*}(x) \leq u(x)$. This taken with the previous inequality shows that $u^{*}(x) \equiv u(x)$ and, being both upper semicontinuous and lower semicontinuous, $u(x)$ is continuous in $R$. 
By using Theorem 2 one can easily see that $u(x)$ is an $\{F\}$-function in $B$.

In Theorem 16 of [1] it is shown that, if $\Omega$ is a bounded open set contained with its boundary $\omega$ in $D$ and if for each $x \in \omega$ there is a circle $\kappa$ such that $\bar{\Omega} \cap \bar{K}=x$, then $\Omega$ is a Dirichlet region for the $\{F\}$-functions. For such a region $\Omega$ we may construct barrier sub-\{F\} functions as was done in Theorem 16 of [1] and thus obtain equality of $u(x)$ and $f(x)$ on the boundary $\omega$ of $\Omega$. This would imply the continuity of $u(x)$ in $\bar{\Omega}$.

8. Proof of Theorem 12. Let $\Omega \subset D$ be a bounded Dirichlet region for the $\{F\}$-functions of the type mentioned in the previous paragraph. By Definition 5 the $\epsilon$-sub- $\{F\}$ function $g(x)$ is bounded on $\bar{\Omega}$ and hence by Postulate 4 and Theorem 9 has a sub- $\{F\}$ minorant in $\Omega$. Since $g(x)$ is upper semicontinuous in $\bar{\Omega}$, there is a decreasing sequence of continuous functions $\left\{f_{n}(x)\right\}$ converging to $g(x)$ in $\bar{\Omega}$. By Theorem $13 f_{n}(x)$ has a maximal sub- $\{F\}$ minorant $u_{n}(x)$ in $\Omega$. The sequence $\left\{u_{n}(x)\right\}$ is uniformly bounded and decreasing in $\bar{\Omega}$ and therefore by Theorem 10 converges to $u(x)$ which is $\operatorname{sub}-\{F\}$ in $\Omega$. Clearly $u(x)$ is the maximal sub- $\{F\}$ minorant of $g(x)$ in $\Omega$.

For each $x \in \bar{\Omega}$, either $u_{n}(x)=f_{n}(x)$ or $u_{n}(x)<f_{n}(x)$. If $u_{n}(x)<f_{n}(x)$, let $\Omega^{\prime}$ be the component containing $x$ of the open subset of $\bar{\Omega}$ in which $u_{n}(x)<$ $f_{n}(x)$. Then $u_{n}(x)$ is an $\{F\}$-function in $\Omega^{\prime}$ and agrees with $f_{n}(x)$ on the boundary of $\Omega^{\prime}$. Hence $g(x) \leq u_{n}(x)$ on the boundary of $\Omega^{\prime}$ and therefore $g(x) \leq \epsilon+$ $u_{n}(x)$ in $\Omega$ : Thus we have

$$
g(x) \leq u_{n}(x)+\epsilon
$$

in $\Omega$ and letting $n$ become infinite

$$
u(x) \leq g(x) \leq u(x)+\epsilon
$$

in $\Omega$.

This proves Theorem 12 for the above class of Dirichlet domains in $D$. Now consider a nested sequence of such bounded Dirichlet domains $\left\{\Omega_{\kappa}\right\}$ exhausting $D$. Let $\left\{u_{\kappa}(x)\right\}$ be the associated sequence of maximal sub- $\{F\}$ minorants of $g(x)$. This serquence is obviously decreasing and, since for $\kappa \geq N$

$$
g(x)-\epsilon \leq u_{\kappa}(x) \leq g(x)
$$

on $\Omega_{N}$, is uniformly bounded on each closed subset of $D$. Another application of Theorem 10 shows that the sequence $\left\{u_{K}(x)\right\}$ converges to a function which is $\operatorname{sub}-\{F\}$ in $D$, is clearly the maximal sub-\{F\} minorant of $g(x)$ in $D$, and 
satisfies the inequality of Theorem 12 in $D$. Theorem 12 is proved.

In a subsequent publication it will be shown that the solutions of certain elliptic partial differential equations satisfy the postulates of the $\{F\}$-functions.

\section{REFERENCES}

1. E. F. Beckenbach and L.K. Jackson, Subfunctions of several variables, Pacific J. Math. 3 (1953), 291 - 313.

2. E. F. Beckenbach, Generalized convex functions, Bull. Amer. Math. Soc. 43 (1937), $363-371$.

3. J.W. Green, Approximately subharmonic functions, Proc. Amer. Math. Soc. 3 (1952), 829-833.

4. D. H. Hyers and S. M. Ulam, Approximately convex functions, Proc. Amer. Math. Soc. 3 (1952), 821 - 828.

UNIVERSITY OF NEBRASKA 


\section{PACIFIC JOURNAL OF MATHEMATICS}

\section{EDITORS}

\author{
H. L. ROYDEN \\ Stanford University \\ Stanford, California \\ E. Hewitt \\ University of Washington \\ Seattle 5 , Washington
}

R. P. Dilworth

California Institute of Technology Pasadena 4, California

A. HorN*

University of California

Los Angeles 24, California

\section{ASSOCIATE EDITORS}

\author{
H. BUSEMANN \\ HERBERT FEDERER \\ MARSHALL HALL
}

\author{
P. R. HALMOS \\ HEINZ HOPF
}

ALFRED HORN
R. D. JAMES

BORGE JESSEN

PAUL LÉVY
GEORGE PÓLYA

J. J. STOKER

KOSAKU YOSIDA

\section{SPONSORS}

UNIVERSITY OF BRITISH COLUMBIA

CALIFORNIA INSTITUTE OF TECHNOLOGY

UNIVERSITY OF CALIFORNIA, BERKELEY

UNIVERSITY OF CALIFORNIA, DAVIS

UNIVERSITY OF CALIFORNIA, LOS ANGELES

UNIVERSITY OF CALIFORNIA, SANTA BARBARA

MONTANA STATE UNIVERSITY

UNIVERSITY OF NEVADA

OREGON STATE COLLEGE

UNIVERSITY OF OREGON

UNIVERSITY OF SOUTHERN CALIFORNIA

\author{
STANFORD RESEARCH INSTITUTE \\ STANFORD UNIVERSITY \\ UNIVERSITY OF UTAH \\ WASHINGTON STATE COLLEGE \\ UNIVERSITY OF WASHINGTON
}

AMERICAN MATHEMATICAL SOCIETY HUGHES AIRCRAFT COMPANY SHELL DEVELOPMENT COMPANY

Mathematical papers intended for publication in the Pacific Journal of Mathematics should be typewritten (double spaced), and the author should keep a complete copy. Manuscripts may be sent to any of the editors. Manuscripts intended for the outgoing editors should be sent to their successors. All other communications to the editors should be addressed to the managing editor, Alfred Horn at the University of California, Los Angeles 24, California.

50 reprints of each article are furnished free of charge; additional copies may be obtained at cost in multiples of 50 .

The Pacific Journal of Mathematics is published quarterly, in March, June, September, and December. The price per volume (4 numbers) is $\$ 12.00$; single issues, $\$ 3.50$. Back numbers are available. Special price to individual faculty members of supporting institutions and to individual members of the American Mathematical Society: $\$ 4.00$ per volume; single issues, $\$ 1.25$.

Subscriptions, orders for back numbers, and changes of address should be sent to Pacific Journal of Mathematics, c/o University of California Press, Berkeley 4, California.

Printed at Kokusai Bunken Insatsusha (International Academic Printing Co., Ltd.), No. 10, 1-chome, Fujimi-cho, Chiyoda-ku, Tokyo, Japan.

* During the absence of E. G. Straus.

PUBLISHED BY PACIFIC JOURNAL OF MATHEMATICS, A NON-PROFIT CORPORATION COPYRIGHT 1955 BY PACIFIC JOURNAL OF MATHEMATICS 


\section{Pacific Journal of Mathematics}

\section{Vol. 5, No. $2 \quad$ October, 1955}

Leonard M. Blumenthal, An extension of a theorem of Jordan and von

Neumann ........................................ 161

L. Carlitz, Note on the multiplication formulas for the Jacobi elliptic functions.......................................... 169

L. Carlitz, The number of solutions of certain types of equations in a finite

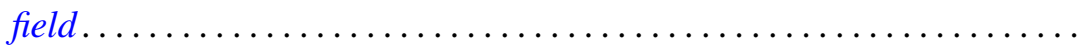

George Bernard Dantzig, Alexander Orden and Philip Wolfe, The generalized simplex method for minimizing a linear form under linear

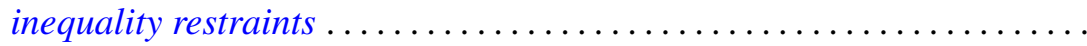

Arthur Pentland Dempster and Seymour Schuster, Constructions for poles and polars in n-dimensions . . . . . . . . . . . . . . . . . . . . 197

Franklin Haimo, Power-type endomorphisms of some class 2 groups ...... 201

Lloyd Kenneth Jackson, On generalized subharmonic functions ......... 215

Samuel Karlin, On the renewal equation ...................... 229

Frank R. Olson, Some determinants involving Bernoulli and Euler numbers of higher order................................ 259

R. S. Phillips, The adjoint semi-group ........................ 269

Alfred Tarski, A lattice-theoretical fixpoint theorem and its applications ... 285

Anne C. Davis, A characterization of complete lattices .............. 311 